\title{
L'intervieweur face au discours littéraire : stratégies de positionnement chez Madeleine Chapsal, Jacques Chancel et Bernard Pivot
}

The Interviewer and Literary Discourse: Madeleine Chapsal's, Jacques Chancel's and Bernard Pivot's Positioning Strategies

David Martens et Christophe Meurée

\section{(2) OpenEdition Journals}

\section{Édition électronique}

URL : http://journals.openedition.org/aad/1639

DOI : 10.4000/aad. 1639

ISSN : 1565-8961

\section{Éditeur}

Université de Tel-Aviv

\section{Référence électronique}

David Martens et Christophe Meurée, «L'intervieweur face au discours littéraire : stratégies de positionnement chez Madeleine Chapsal, Jacques Chancel et Bernard Pivot », Argumentation et Analyse du Discours [En ligne], 12 | 2014, mis en ligne le 20 avril 2014, consulté le 23 septembre 2019. URL : http://journals.openedition.org/aad/1639; DOI : 10.4000/aad.1639

Ce document a été généré automatiquement le 23 septembre 2019.

\section{cc)}

Argumentation \& analyse du discours est mis à disposition selon les termes de la licence Creative Commons Attribution - Pas d'Utilisation Commerciale - Pas de Modification 4.0 International. 


\section{L'intervieweur face au discours} littéraire : stratégies de positionnement chez Madeleine Chapsal, Jacques Chancel et Bernard Pivot

The Interviewer and Literary Discourse: Madeleine Chapsal's, Jacques Chancel's and Bernard Pivot's Positioning Strategies

David Martens et Christophe Meurée

\section{NOTE DE L'AUTEUR}

La présente étude a été réalisée dans le cadre des recherches du groupe MDRN (www.mdrn.be) de l'Université de Louvain (KU Leuven). MDRN conduit, pour la période 2011-2015, une Action de Recherche Concertée (Literature and its Multiple Identities 1900-1950) financée par le Conseil de Recherche de la KU Leuven et coordonne le Pôle d'attraction interuniversitaire Literature and Media Innovations (lmi.arts.kuleuven.be), financé par la Politique scientifique fédérale belge (www.belspo.be), auquel participe le Centre de Recherche sur l'Imaginaire (CRI) de l'Université catholique de Louvain (Louvain-la-Neuve). L'étude des entretiens d'écrivain constitue l'un des axes de recherche commun à ces deux programmes.

1 Selon un stéréotype assez largement partagé, la pratique de l'entretien semble n'accorder qu'une place secondaire à l'intervieweur. Le rôle qui lui est assigné au sein de l'échange verbal tend fréquemment à passer pour anecdotique. De ce point de vue, l'intervieweur ne serait qu'un chaînon dont la fonction vise à favoriser la médiation, $a$ priori le plus invisible possible, en vertu du principe d'effacement du dispositif médiatique (Bolter et Grusin 1999) et du médiateur qui en constitue l'un des 
paramètres principaux. L'intervieweur apparaît à cette aune comme le scotome de la scénographie de l'entretien, au sein de laquelle il se bornerait à recueillir la parole de la personne interrogée, tant et si bien que son travail pourrait être réalisé par un(e) autre sans que la donne en soit fondamentalement changée.

Or, le discours de l'entretien se fonde sur une co-construction (Yanoshevsky 2011). Par ses interventions et les réponses fournies, l'intervieweur exerce de facto un impact sur le discours de son interlocuteur dans la mesure où celui-ci, acceptant de se prêter au jeu du dialogue, répond en fonction des questions qui lui sont adressées - toute entorse à ce paramètre du «contrat interactionnel » qui définit l'espace de l'entretien (Charaudeau 1989 : 15) se conçoit comme une rupture, plus ou moins ludique, avec l'un des attendus formels (l'alternance des questions et des réponses) qui caractérise le genre. Responsable de la tenue de l'échange verbal et en vertu des fonctions qu'il y assume, l'intervieweur peut dès lors être considéré comme l'auteur au même titre quoique sur un autre plan - que son ou ses interlocuteurs (pour le $19^{\mathrm{e}}$ siècle, voir Seillan 2010 : 1028-1030). Ainsi certains intervieweurs ont-ils pu devenir des figures en vue du monde médiatique, à l'instar de Madeleine Chapsal, Jacques Chancel ou Bernard Pivot, dont on lit, écoute ou regarde les entretiens parfois indépendamment de l'intérêt que l'on porte à leurs invités.

Dans la conduite des dialogues, chaque intervieweur compose un ethos singulier (Amossy 2010), qui tient notamment à une façon particulière de se positionner par rapport aux types de discours en jeu. L'entretien suppose en effet une négociation qui touche non seulement aux modalités de l'interaction verbale (Kerbrat-Orecchioni 2005) mais aussi aux enjeux qui découlent de la rencontre entre différents types de discours, en l'occurrence les discours journalistique et littéraire ${ }^{1}$. Relevant a priori du journalisme, le genre de l'interview permet l'interaction de deux types de discours au sein d'un domaine particulier du champ. En pareil contexte, le journaliste joue certes sur son terrain - la presse pour Chapsal, la radio pour Chancel et la télévision pour Pivot - mais se trouve conduit, afin de répondre aux attentes du public, à offrir un espace (et un temps) à la parole d'un interlocuteur qui représente un autre type de discours, dont les impératifs imprègnent forcément sa pratique. Comment, dès lors, l'intervieweur négocie-t-il cette double participation qui caractérise sa position au sein de l'hétérogénéité discursive qui sous-tend fréquemment l'entretien?

\section{Une hétérogénéité discursive contrainte}

\subsection{L'hétérogénéité discursive d'un genre}

4 Au sein de ce genre particulier que constitue l'entretien, les interlocuteurs dialoguent en vertu d'une subjectivité singulière, mais aussi en fonction de leur appartenance à des champs discursifs potentiellement distincts amenés à interagir à la faveur de l'entretien. Forme phare de la reconfiguration du système des genres discursifs qu'a impliqué l'émergence de la "matrice médiatique» (Thérenty 2007) liée à la « civilisation du journal » (Kalifa et al. 2010), l'entretien apparaît comme un des lieux de rencontre entre deux interlocuteurs et deux espaces discursifs au moins: le journalistique, le plus souvent, et, selon le titre au regard duquel l'interviewé est interrogé, d'autres types de discours (politique, religieux, littéraire, etc.). Le genre 
s'institue ainsi comme un espace d'interactions entre discours, soumis à plusieurs types de contraintes.

\subsection{Un espace interactionnel contraint}

5 Galia Yanoshevsky [à paraître]) $)^{2}$ souligne combien le genre de l'entretien implique un « système complexe et en interaction de rôles sociaux établis à travers des pratiques discursives et modifiés dans l'échange à travers des processus de négociation ». Ces derniers, résultant notamment de l'interaction entre les champs discursifs mis en relation dans l'entretien, se configurent en fonction de deux paramètres au moins, dont les effets se conjuguent : d'une part, la situation énonciative spécifique de l'entretien, en particulier les modalités de la relation entre les différents champs de discours impliqués; d'autre part, le rôle que joue le médium (qu'il s'agisse d'une forme écrite périodique ou livre -, de la radio ou de la télévision) dans la réalisation de l'échange, et, partant, le positionnement de l'intervieweur.

\subsubsection{Entre « espace d'étayage » et « espace associé »}

Selon les domaines en présence dans la rencontre entre types de discours qui participe de la "composante interactionnelle» (Charaudeau 1989: 15) régissant l'échange, l'intervieweur ne se positionne pas exactement de la même façon par rapport à son interlocuteur et au champ discursif qu'il représente. Alors que, de manière générale, l'éthique professionnelle des intervieweurs les conduit fréquemment à pousser leurs interlocuteurs dans leurs derniers retranchements, face à un écrivain, l'intervieweur occupe plutôt une position de faire-valoir. Face au discours littéraire, le genre relève d'une interaction entre, du côté de l'intervieweur, la part de l'« espace d'étayage " (Pascale Delormas ${ }^{3}$ ) qui échappe à l'auteur et, s'agissant de l'écrivain, l'« espace associé », qui comprend les "textes d'auteur qui accompagnent [s]es œuvres» (Maingueneau 2004: 113) et qui se trouvent (censément) au service de "l'espace canonique $»^{4}$ que constituent ces dernières. La confrontation de deux auctorialités (Martens et Meurée [à paraître]) que génère l'entretien trouve là un point de friction potentiel qui dépend des modes de relation entre les espaces institutionnels et discursifs interagissant. C'est en fonction de cette interaction, hiérarchisée et déterminée par une aspiration à approcher l'espace canonique qu'incarne l'écrivain, que l'intervieweur est conduit à se positionner.

\subsubsection{L'intervieweur et le médium}

$7 \quad$ La place occupée par l'intervieweur dans le discours se trouve également conditionnée par le contexte du médium dans lequel l'entretien est réalisé et par lequel il est diffusé. En l'occurrence, la presse écrite, la radio et la télévision ne présentent pas les mêmes implications, tant à l'endroit de l'entretien comme genre discursif que pour ce qui touche à son traitement du discours littéraire et des écrivains. Ainsi, sur le plan pragmatique des conditions de réalisation matérielle des entretiens, le direct qu'imposent certaines émissions radiophoniques ou télévisuelles n'est pas de mise pour les échanges destinés à revêtir une forme écrite. Les conditions de réalisation de l'entretien informent en effet la conduite singulière du dialogue. En outre, l'histoire de chaque médium le situe dans une relation spécifique avec le discours littéraire. Tout 
intervieweur configure ainsi son ethos discursif, non seulement en fonction des rapports entre le discours journalistique et le discours littéraire, mais aussi de ceux qu'entretient la littérature avec le médium qui offre le cadre de l'entretien. Enfin, il convient de ne pas négliger les reprises d'entretiens, notamment en volume, ainsi que les discours qui les accompagnent. Ils sont souvent l'occasion pour les intervieweurs de revenir sur leur pratique à travers un médium, le livre, qui confère une nouvelle portée à leur travail, en particulier au regard de sa relation à l'espace littéraire.

\subsection{Devant le discours littéraire}

8 Tout intervieweur négocie de façon singulière ses interactions avec les représentants d'autres types de discours. Dès lors qu'il s'agit d'interroger des écrivains, acteurs majeurs du champ discursif spécifique qu'est la littérature, la parole de l'intervieweur se constitue en fonction de ce que le littéraire représente de façon particulière au regard de la pratique de l'entretien et de son ancrage journalistique. Le positionnement qui en résulte, bien qu'il puisse varier selon l'environnement médiatique et les individus en présence, modèle la singularité des intervieweurs. En témoignent les entretiens de trois intervieweurs spécialisés dans le domaine culturel, parmi les plus célèbres de la seconde moitié du $20^{e}$ siècle en France: Madeleine Chapsal, Jacques Chancel et Bernard Pivot ont en effet manifesté des affinités particulières avec la littérature, qui les ont même amenés à taquiner la muse à leur tour.

Entre quête de proximité et prise de distance avec l'espace canonique du discours littéraire, le spectre des attitudes et des pratiques semble particulièrement diversifié : Chapsal affiche une accointance forte avec la littérature et les écrivains, au point de neutraliser son statut de journaliste au profit de celui d'écrivain; Chancel témoigne d'une position intermédiaire qui, si elle se caractérise par une indéniable attraction envers le discours littéraire, n'éloigne cependant jamais le journaliste du métier qu'il exerce ; Pivot assume quant à lui pleinement sa profession, en l'agrémentant d'apprêts et d'appas empruntés au littéraire. Ces stratégies de positionnement se traduisent dans la réalisation et la diffusion des entretiens, de même qu'à travers les discours rétrospectifs de ces intervieweurs sur leur pratique.

\section{Madeleine Chapsal ou comment n'être pas journaliste}

À travers les entretiens qu'elle a publiés de 1959 à 1978 dans L'Express, Madeleine Chapsal s'est acquis une réputation à part dans le monde des intervieweurs culturels en France, tout en menant parallèlement une œuvre d'écrivain. Au sein de ce périodique à vocation généraliste, ces entretiens appartiennent à la rubrique dévolue à l'actualité littéraire. Pour autant, en dépit de l'espace spécifique qu'il consacre à la littérature, cet environnement médiatique ne ressortit pas au discours littéraire stricto sensu, pas plus que l'entretien ne relève du système des genres littéraires institués ni même, a priori, de la littérature - soit de l'espace canonique - sinon moyennant le recours à des marqueurs de littérarité (voir Martens et Meurée [à paraître]). 


\subsection{Des écrivains bien curieux}

11 Chapsal incarne le versant journalistique de la rencontre et représente le périodique aussi bien que son lectorat. Cependant, la configuration de ses entretiens tend régulièrement à remettre en question le rôle de l'intervieweuse, ainsi que son statut au regard des deux espaces discursifs dont l'entretien assure la rencontre. À de multiples reprises, les dialogues se caractérisent en effet par une inversion des rôles: alors qu'elle est chargée de poser les questions, Chapsal se retrouve interrogée par ses interlocuteurs. Si le fait n'est pas foncièrement étrange en régime conversationnel, notamment en raison des négociations propres aux interactions verbales, ces interversions frappent toutefois par leur récurrence dans le cadre d'un discours aussi formellement contraint que l'entretien.

12 Le phénomène se présente avec une intensité remarquable dans l'entretien accordé par Saint-John Perse. Le récipiendaire du Prix Nobel de littérature pose en effet à son interlocutrice l'intégralité des questions figurant dans le texte publié, qui apparaît davantage comme un récit de rencontre que comme un entretien à proprement parler : " Est-ce que la vie vous a blessée ? fait très mal, vraiment mal ? ", " Savez-vous porter la main aux choses?» ou encore «Aimez-vous les bêtes?» (Chapsal 1960b : 50). Le cas de figure le plus remarquable est celui de Truman Capote qui, à l'entame de l'entretien, endosse de sa propre initiative le rôle d'intervieweur, non pour questionner Chapsal, cette fois, mais bien pour s'interroger lui-même :

En visite à Paris pour la première fois depuis six ans, [Truman Capote] me reçoit dans un salon désert de l'hôtel Ritz, près d'une véranda sur laquelle coule la pluie d'avril - cadre parfait pour une nouvelle de Truman Capote...

Truman Capote : - Voulez-vous que je m'interroge moi-même?

- Je vous en prie.

T. Capote - «M. Truman Capote, que faites-vous en ce moment? Eh bien, je travaille sur [...] un livre qui n'est pas un roman, mais un reportage. Le sujet en est un crime qui s'est passé dans une minuscule ville du Kansas [...]. [...] Cela s'appellera In cold blood [...].

Voilà, c'est tout pour ma littérature. Quoi d'autre?» (Chapsal 1962 : 32-33)

13 La fréquence de ces interversions témoigne de la souplesse que Chapsal entend imprimer à son mode opératoire et aux fonctions dévolues aux interlocuteurs. Tout se passe comme s'il s'agissait de fragiliser la ligne de démarcation qu'instaure le cadre de l'entretien en termes de rôles discursifs, au profit d'une familiarité pleinement réalisée. Dans le cas de Capote, l'entretien s'ouvre de façon suggestive comme une fiction de l'auteur interviewé, la scène s'inscrivant au sein d'un espace présenté comme typique de l'univers de l'auteur, selon une topique du genre (Seillan 2002: 44). La rencontre s'opère sur le terrain de l'écrivain qui, certes, s'improvise temporairement journaliste, mais sur un mode parodique qui estompe d'autant mieux la frontière séparant les discours journalistique et littéraire.

\subsection{Le secret de l'écrivain}

14 L'interpénétration du journalistique et du littéraire en passe également par l'attitude de Chapsal envers les écrivains. En 1959 déjà, son entretien avec Jean-Paul Sartre le démontre exemplairement. L'intervieweuse y expose comment l'auteur, en pleine répétition des Séquestrés d'Altona, accorde quelques instants à certains "journalistes » qui «l'attendent » : «J.-P. Sartre va s'entretenir quelques instants avec les envoyés de 
France-Soir, du Figaro, de France-Observateur ». Ce n'est que dans un second temps qu'il se consacre à Chapsal : "Sartre en a fini avec les journalistes. Il m'entraîne dehors, à la terrasse d['un] café » (Chapsal 1959 : p. 27). La position adoptée rompt avec son statut de journaliste représentant L'Express. Davantage, la nature même de la rencontre se voit révoquée lorsque, au terme de l'échange, Sartre déclare à son interlocutrice : «Il faut que nous reparlions de tout cela. J'ai beaucoup de choses à vous dire à propos du théâtre. Nous pourrions faire un "entretien" ", comme si ce que le lecteur achève alors de lire n'en était pas tout à fait un.

Cette discrimination entre la position de Chapsal et ce qu'elle présente sous l'aspect d'un journalisme grégaire contribue à mettre en place un ethos discursif particulier : il s'agit de s'exposer comme une intervieweuse aux antipodes du stéréotype journalistique. Elle accentue d'ailleurs de façon manifeste cette stratégie de positionnement à l'occasion de la reprise de ses entretiens en volumes : Les Écrivains en personne (1960) et Quinze écrivains (1963) parus chez Julliard, Envoyez la petite musique (Grasset, 1983) et Ces voix que j'entends encore (Fayard, 2011). Dans les textes qui présentent a posteriori ses entretiens dans ces ouvrages, Chapsal marque plus que jamais ses distances avec le monde journalistique. Le récit de sa rencontre avec Borges (L'Express, 21 février 1963) est sans conteste le plus significatif à cet égard. Elle s'y distingue résolument du groupe qui gravite autour de l'écrivain :

Quand j'arrivai, j'eus la surprise de trouver Borges assis sur un vaste fauteuil au milieu du hall de l'hôtel, comme un prince sur son trône. Des journalistes à l'air grave faisaient la queue pour obtenir, à tour de rôle, le droit de lui parler quelques instants.

[...] Travailler en public, comme à une conférence de presse, n'était pas la condition idéale pour conduire un dialogue intime ! J'eus envie de m'en aller...

La curiosité me retint. C'était la première fois qu'il m'était donné de voir fonctionner des confrères face à un écrivain. Ceux-là étaient impressionnants de sérieux ! Je tendis l'oreille.

« N'est-ce pas, disait à Borges le journaliste assis près de lui, que c'est bien ce que vous avez voulu dire dans Fictions? »

[...]

L'écrivain, lui, hochant la tête, comme je l'avais vu faire au pape en audience publique à chacun de ceux qui s'inclinaient devant lui pour solliciter une grâce, sa bénédiction... Le journaliste se leva, radieux, et regarda d'assez haut la cohorte de ses concurrents!

"Vous voyez, semblait-il dire, aucun de vous n'est aussi proche que moi du "secret" de Borges!» (Chapsal 1984 : 275-276)

Si Chapsal présente les autres intervieweurs comme des "confrères", toute la scène d'énonciation concourt à l'élever en figure d'exception. La relation qu'elle désire instaurer avec l'écrivain, en position de majesté et de sacralisation (« comme un prince sur son trône ", "pape ", " grâce ", " bénédiction "), ne s'ancre pas dans une lecture pédante de l'œuvre dont la vocation première semble de damer le pion aux autres journalistes. La scène dépeint le jeu de concurrence entre " confrères ", résultant de la volonté d'établir et de manifester fièrement une familiarité unique avec le «secret » de l'écrivain, qui confère une aura de mystère à l'espace canonique mobilisé, en même temps que sa valeur à l'entretien. Mais alors que, dans l'entretien auquel elle assiste, Borges se voit constamment interrompu et par conséquent empêché de s'exprimer, Chapsal rêve pour sa part d'un "dialogue intime " qui la distinguerait de ceux qui, précisément, entendent se distinguer. 
Quand vint mon tour, je m'approchai doucement du poète aveugle, et, touchée par son regard vide [...], je commençai par l'interroger sur son voyage. Je voulais qu'il sache, au son de ma voix, qu'il avait affaire à une femme, guère trop savante...

Puis je lui posai des questions simples, concrètes, dont je savais qu'un esprit complexe et raffiné comme le sien saurait faire sa proie : «Qu'est-ce qu'écrire?", "Comment écrivez-vous?»

Un murmure de surprise, puis des ricanements s'élevèrent dans mon dos. Les

« borgesiens » n'en revenaient pas qu'on osât traiter ainsi leur Borges.

[...]

Loin d'être choqué, Borges me répondit avec simplicité et même il s'anima. (Chapsal

$1984: 275-277$, passim)

Dans son récit, Chapsal convoque plusieurs valeurs cardinales du genre (intimité, simplicité) en les confrontant à d'autres (distance critique et prétention à la connaissance, complexité), qui se situent aux antipodes de la conception de l'entretien qu'elle promeut. La formule "guère trop savante", relativement ambiguë dans la mesure où elle conjugue euphémisme et hyperbole, mobilise les facettes de la féminité et d'une certaine ignorance iconoclaste autant qu'elle pointe ironiquement l'excès de savoir d'un "confrère " qui ne laisse guère de place à l'écrivain lui-même et dont l'« entretien", de ce fait, perd toute valeur. La simplicité qui consiste à interroger Borges à propos de son voyage apparaît par contraste comme une façon d'introduire de la convivialité en accordant une préséance à la personne au détriment de la fonction pour laquelle elle est sollicitée dans le cadre de l'entretien. Cette simplicité permet d'installer une proximité de l'intervieweuse avec le maître et l'espace canonique qu'il incarne. Le geste de désacralisation pousse le grand homme à répondre simplement à son tour et, ce faisant, à "s'anim[er] », c'est-à-dire, littéralement, à (re)prendre vie. L'entretien n'apparaît plus comme le fait d'un agent de l'espace d'étayage (le journaliste) qui s'efforce de s'élever à la hauteur du représentant de l'espace canonique (l'écrivain), au point de ne plus lui laisser voix au chapitre ; désormais, c'est l'écrivain en personne qui parle.

\subsection{Un écrivain en personne}

Avec Sartre ou avec Borges, le positionnement de Chapsal repose sur une paratopie (Maingueneau 2004) qui se traduit aussi bien dans le jeu des questions que dans la constitution d'un ethos singulier. Sa stratégie permet à cette journaliste qui n'en est pas tout à fait une de gagner sur deux tableaux (ou champs discursifs). D'une part, suscitant chez l'écrivain un désir de parler, l'intervieweuse mobilise une autre valeur cardinale du genre, le savoir-faire-dire ; corollairement, grâce à la proximité qu'elle instaure, Chapsal rapproche ses lecteurs d'un espace canonique perçu (et scénographiquement posé) comme difficilement accessible. D’autre part, en dépit de la part de modestie qui sous-tend l'ethos de l'intervieweuse, l'instauration d'une familiarité libère Chapsal de l'étiquette journalistique. Ces scènes d'énonciation la font participer du statut d'écrivain de ses interlocuteurs, qu'elle acquerra effectivement par l'œuvre littéraire qu'elle entreprend au début des années 1970.

19 Si ce type de gestion de la rencontre avec le littéraire opère dès les entretiens tels qu'ils ont été publiés dans L'Express, il semble que la dynamique se renforce dans le geste de colliger les dialogues au sein de recueils, qui consolide l'auctorialité de l'intervieweuse. Elle signe ces livres successifs comme des ouvrages propres. Si l'usage est assez courant pour le livre d'entretiens, il n'en atteste pas moins d'une appropriation contribuant à 
faire de Chapsal une auteure à part entière, via un espace livresque qui demeure le moyen de consécration privilégié de l'écrivain (Maingueneau 2009). La fonction symbolique du livre semble confirmée par la façon dont l'homme de radio qu'est Jacques Chancel l'exploite lorsque, à son tour, il rassemble en volumes une sélection de ses entretiens, dans lesquels il manifeste une inclination particulière pour le littéraire, quoique plus réservée que celle de Chapsal.

\section{Jacques Chancel ou les « épousailles » de la littérature}

Diffusée en direct sur France-Inter, Radioscopie s'est distinguée en France, de 1968 à 1982, puis à nouveau à partir de $1988^{5}$, comme l'émission d'entretiens radiophoniques de référence. La série a permis à Jacques Chancel d'imposer une manière singulière, combinant une courtoisie bienveillante destinée à mettre à l'aise ses invités et une volonté de ne pas écarter les sujets épineux, et caractérisée par une propension aux interrogations fondées sur des alternatives, ainsi que par un goût pour les grandes questions anthropologiques, sociales et philosophiques (la vie, la mort...). Devant le succès de l'émission, celui qui est devenu, au fil des ans, une vedette du paysage audiovisuel hexagonal, a été amené à rassembler, en plusieurs volumes parus chez Robert Laffont entre 1970 et 1976, les entretiens qui, indique-t-il dans l'« Avantpropos » au premier tome de ces Radioscopies écrites, «ont suscité le plus de courrier » (Chancel 1970 : 11) à la suite de leur diffusion.

\subsection{Incursions du littéraire}

Compte tenu de la nature du médium radiophonique, les conditions de gestion de la part du littéraire dans l'espace discursif diffèrent de celles qui régissent une rubrique dans un hebdomadaire généraliste. La différence entre l'oral et l'écrit fait de la radio un environnement médiatique moins spontanément associé à la création littéraire, dont le support privilégié demeure le livre. La programmation de Radioscopie ne permet pas de la dénombrer parmi les émissions littéraires. Au sein du panel varié (acteurs, hommes de loi, médecins, hommes politiques, etc.) de figures publiques «radioscopées » par Chancel (plus de 2500 émissions), les écrivains sont certes bien représentés, mais ne sont cependant pas majoritaires.

En vertu de la " composante situationnelle » (Charaudeau 1989:15) de l'émission, le but de Chancel est de mener à bien un dialogue adressé à un public potentiellement très divers, qui écoute l'émission au moment où elle a lieu (ou qui lit l'entretien après coup). L'animateur doit l'intéresser à des personnalités elles-mêmes très différentes, tout en négociant la rencontre du discours journalistique avec des représentants d'autres types de discours et/ou de pratiques. De ce fait, rien d'étonnant à ce que l'identité compte parmi les préoccupations systématiquement au cœur du dispositif énonciatif mis en œuvre par l'intervieweur. Il s'adonne en effet, à l'entame de presque tous ses entretiens, à un portrait de son invité, brossé en quelques traits, qu'il ponctue très souvent d'une question portant explicitement sur un aspect particulier de l'identité de son interlocuteur.

Du point de vue pragmatique, de telles amorces - qu'illustre parfaitement la première question à Brigitte Bardot : «Brigitte Bardot, est-il difficile, non pas d'être Bardot, mais 
de le rester?» (Radioscopie, 7 février 1970) - permettent à Chancel d'expliciter les motivations de l'entretien - une actualité, la plupart du temps - et de poser les bases, informatives et thématiques, d'un certain nombre de questions qui vont suivre. À cet égard, s'il est légitime d'entendre l'intervieweur parler de littérature en compagnie d'écrivains, la chose parait plus surprenante avec des hommes de science ou de loi. Les nombreuses allusions littéraires qui émaillent les dialogues de Chancel avec des interlocuteurs qui ne sont pas des écrivains interpellent moins par leur relative fréquence que par le caractère parfois abrupt de leur intrusion dans le cours de la conversation. Chancel demande ainsi à Jean Bernard, au cours d'une séquence ayant trait à la façon dont le médecin envisage la mort : «Pour bien comprendre la vieillesse, faut-il avoir lu Charles Péguy et Simone de Beauvoir?» (Radioscopie, 24 janvier 1973). Son interlocuteur lui répond en ramenant l'échange sur le terrain de sa compétence médicale. Il neutralise ainsi la prédominance que l'intervieweur semble accorder au littéraire et que Bernard assimile au philosophique: "Je pense que cela aide à la comprendre, mais les problèmes posés en médecine sont un peu différents de ceux évoqués par les philosophes » (ibid.).

\subsection{Portraits d'écrivains} sujets abordés, affectent sensiblement la teneur du discours. Plus fondamentalement, elles infléchissent les portraits que l'animateur de Radioscopie s'emploie à proposer à ses auditeurs. Ainsi Chancel amène-t-il fréquemment ses invités à considérer certaines de leurs vies parallèles, avortées, désirées, ou en instance de prendre forme, aspects biographiques le plus souvent méconnus du public. Si les entames des entretiens se fondent le plus souvent sur ce qui a rendu l'interlocuteur célèbre, l'intervieweur en retouche régulièrement les portraits en leur découvrant, voire en leur conférant une dimension littéraire, jusqu'à faire de ses invités rien de moins que des écrivains en puissance.

Après que Jean Bernard lui a affirmé que "[1]orsqu'on est médecin, on s'exprime complètement dans son acte de médecin ", Chancel place cette profession de foi sous le signe de la littérature (et de la philosophie, intégrant ainsi l'équivalence précédemment établie par son interlocuteur) en lui rétorquant manifestement sur un mode interrogatif : "Oui, mais lorsque vous êtes médecin, vous oubliez un peu ce que vous avez été, c'est-à-dire poète... » (ibid.). Certes, cet ancien Résistant appelé à entrer à l'Académie française en 1975 au fauteuil de Marcel Pagnol est l'auteur d'un recueil de poèmes, Survivance, publié en 1944. Chancel se borne en ce sens à réaliser son travail de journaliste, à savoir délivrer un portrait qui n'omette aucune facette du modèle. Dans le même temps, le portrait composé par le journaliste tend à tracer la silhouette d'une figure d'écrivain quand son vis-à-vis entend manifestement ne s'exprimer qu'en sa qualité de médecin 6 .

De façon analogue, Chancel déclare à Albert Naud, qui a défendu Céline lors de ses démêlés avec la justice après 1945 et qui a lui-même tâté de la plume : "Maître Albert Naud, vous êtes un grand avocat, mais est-ce que vous ne voudriez pas aujourd'hui être seulement un homme de lettres ? (Radioscopie, 12 novembre 1969). Manière de réduire ce pour quoi son interlocuteur est alors célèbre au profit d'une part moins connue de son activité publique. Moins radicalement mais plus inopinément, Chancel invite

Argumentation et Analyse du Discours, 12 | 2014 
Jacques Monod à envisager de quitter son champ de compétences pour embrasser celui de la littérature de fiction :

J.C. - Jacques Monod, est-ce que vous pourriez sortir de votre propre domaine ? Là, vous venez de publier Le hasard et la nécessité, et c'est quand même votre domaine. Vous pourriez écrire un roman?

J.M. - Un roman ? Sûrement pas, non!

J.C. - Vous avez quand même le goût de l'écriture...

J.M. - Ah, beaucoup !

J.C. - Et ça se sent.

J.M. - J'aime écrire, oui. Mais un roman, certainement pas, non... un conte philosophique, peut-être... Une pièce de théâtre, peut-être. Un roman, c'est trop difficile...

J.C. - Le succès de ce livre, c'est un encouragement pour vous, à continuer à écrire ?

(Radioscopie, 18 novembre 1970)

Pareille remarque semble être une véritable incitation. Une aspiration constante semble inciter Chancel à mobiliser la littérature pour orienter le portrait de ses interlocuteurs, quitte à les ériger en écrivains. Le fait est si fréquent que l'on peut se demander s'il n'en va pas d'une inclination de l'intervieweur lui-même.

\subsection{Le marié de la littérature}

Lors de la parution de ses entretiens en volumes, Chancel se livre à une opération consistant, d'une part, à atténuer leur dimension journalistique et, d'autre part, à revendiquer pour eux un statut littéraire. Dans l'avant-propos du premier tome, il place ces dialogues sous le signe de l'intimité et de la bienveillance. Les présentant comme des rencontres « [e]ntre gens de bonne compagnie », « au coin du feu » et marquées par un "climat d'amicale courtoisie " (Chancel 1970 : 12), il déclare avoir « la faiblesse de croire que [s]es interlocuteurs ont vite oublié le micro au cours de ces soixante minutes » (ibid.). Le prétendu oubli du micro constitue un topos du genre : neutralisant l'objet incarnant le médium (et, par métonymie, le studio d'enregistrement aussi bien que l'institution de la radio), l'animateur donne corps à un fantasme de familiarité, celui d'une conversation prétendument naturelle, d'autant plus intéressante qu'elle paraît n'avoir pas été conçue pour l'auditeur, qui voit ainsi flattée sa pulsion non pas voyeuriste mais auditrice.

L'idée que le dialogue ait eu lieu exempt de micro instaure une fiction censément partagée par l'interlocuteur de Chancel comme par les lecteurs des retranscriptions. Par la neutralisation du micro et le motif convoqué du « coin du feu », il fait vaciller la hiérarchie qu'implique la différence de statut des interlocuteurs. Corollairement, il use d'une terminologie qui, en français, tend à tenir l'interview à distance de l'espace littéraire, au profit d'un désignateur plus favorable sur le plan de la valeur esthétique. Ainsi écrit-il dans l'avant-propos du deuxième tome des Radioscopies, alors que celui-ci rassemble des interviewés dont un seul (Roger Peyrefitte) est écrivain: "Onze personnalités se retrouvent sous cette couverture »; elles "furent des interlocuteurs valables» et "deviennent ici mes associés en littérature» (Chancel 1971: 8, nous soulignons).

Quelques années plus tard, Chancel se livre à quelques aveux surprenants au sujet de son émission. Quoique celle-ci l'ait vu accueillir des personnalités de domaines divers, l'intervieweur ne parle que d'écrivains, confessant n'avoir été essentiellement intéressé que par la rencontre avec ce type d'invités. 
À dire vrai, [...] je n'ai voulu Radioscopie que pour combler un vide, apaiser une gourmandise, reconnaître des visages, ceux de l'écrivain, et les accorder aux mots, aux musiques qui rythmaient mon enfance lavedanaise, loin des chapelles où trônaient déjà les princes de l'écriture. Je ne pouvais pas prévoir ce qui m'arriverait mais inconsciemment [...], il ne fait pas de doute que je me préparais aux épousailles - ce tête-à-tête quotidien. Comment aurais-je pu imaginer qu'un jour je partagerais des heures avec Montherlant, Caillois, Barthes, Sartre, Céline, Jouhandeau, Delteil le saint, Malraux, Cocteau (en dehors de toute radio), Cohen, Yourcenar, Borgès et les autres! L'incroyable peut arriver [...]. Tout vient à celui qui n'attend pas. (Chancel $1983: 16-17)$

31 Cette réduction de Radioscopie à une émission littéraire traduit l'attraction exercée par l'espace canonique sur le discours de l'intervieweur. Elle se double en effet d'une sacralisation par la bande des principales figures de l'espace littéraire, les écrivains. Après avoir évoqué les «chapelles où trônaient les princes de l'écriture » à titre de repoussoir, en désignant une valeur littéraire tenue pour factice (le topos du parisianisme), Chancel évoque son propre travail comme une relation à la faveur de laquelle se conjuguent l'intime et le sacré (" épousailles »). Comment s'étonner dès lors que la seule caractérisation qu'il formule pour désigner l'un des auteurs de sa liste aille à Delteil - le "saint" - et que la seule mention individualisant un autre écrivain (Cocteau) consiste à préciser qu'il a partagé avec lui des moments « en dehors de toute radio ", selon une formule qui résonne comme une déclinaison de l'absence de tout micro...

Alors même que sa démarche diffère de façon notable de celle de Madeleine Chapsal, le positionnement de Chancel par rapport au discours littéraire présente certaines analogies avec celui de l'intervieweuse de L'Express. Il se fonde en effet sur une sanctification qui en passe, comme chez Chapsal, par une revendication de simplicité et de familiarité, vertus fréquentes dans la constitution de l'ethos des intervieweurs d'écrivains. Ne cherchant pas à atteindre le saint des saints, Chancel adopte une position paratopique - dont participe une origine provinciale qui apparaît comme un gage d'authenticité - au regard de ce qui passe pour le cœur de la vie littéraire parisienne. Selon un lieu commun du discours mystique, la rencontre avec l'espace canonique advient d'autant mieux qu'elle n'a pas été volontairement désirée. Une distance demeure toutefois. $\mathrm{Si}$, dans ses livres, Chancel présente les entretiens comme participant du discours littéraire, il ne va pas jusqu'à troquer le statut de journaliste contre celui d'écrivain. En ce sens, il occupe le moyen terme entre le positionnement de Chapsal et celui de l'homme de télévision qu'est Bernard Pivot.

\section{Bernard Pivot ou celui qui n'était pas de la paroisse}

Transfuge de la presse écrite (Le Figaro littéraire puis Le Figaro), Bernard Pivot a, durant près de trente ans, occupé le devant de la scène médiatique télévisuelle consacrée à la littérature (et, plus largement, au domaine du livre). À travers trois émissions - Ouvrez les guillemets (1973-1974), Apostrophes (1975-1990) et Bouillon de culture (1991-2001) -, il a donné à voir "l'image d'un "Monsieur Loyal" persifleur et impertinent, dont la gouaille et la verve bon enfant tranchaient avec le ton habituellement compassé des animateurs d'émissions "littéraires" » (Brasey 1987: 111). Nul doute que Bernard Pivot assume pleinement le ton plutôt populaire qui caractérise son ethos d'intervieweur, en dépit de critiques (Régis Debray et Gilles Deleuze, notamment) relatives à une quête du 
sensationnel et à une dramatisation perçues comme déplacées parce qu'elles dégraderaient l'identité du littéraire.

\subsection{Dramaturgies télévisuelles} animateur qui assure un rôle de "direction d'acteurs" (Brasey $1987: 160-161)$ qui se conjugue avec la part d'improvisation propre à ce type d'émission et aux conditions du direct. La diégèse des émissions tourne autour de plusieurs nœuds dramatiques qui attendent les dénouements que pourront leur apporter les différents invités. Ce n'est pas tant que Pivot se mette à la place du lecteur ignorant mais avide: il impose davantage une façon d'introduire sur son plateau l'atmosphère propre à une œuvre ou à un écrivain afin d'en tirer des révélations qui puissent frapper l'imagination du public. Il s'agit de faire saillir ces informations au travers d'une forme qui se met au service du récit, jusqu'à se construire selon une diégèse spécifique.

Si Pivot (2001 : 131-132) souligne la grande difficulté qu'il y a à interroger un romancier par comparaison à un essayiste - que l'on peut toujours ramener au factuel ou à la logique de sa pensée -, c'est tout de même l'univers du récit (romanesque ou théâtral) qui marque le plus de son empreinte le fonctionnement d'Apostrophes (et de Bouillon de culture). Ainsi Pivot suggère-t-il, dans son entretien avec Marguerite Yourcenar, une suite possible au roman Alexis :

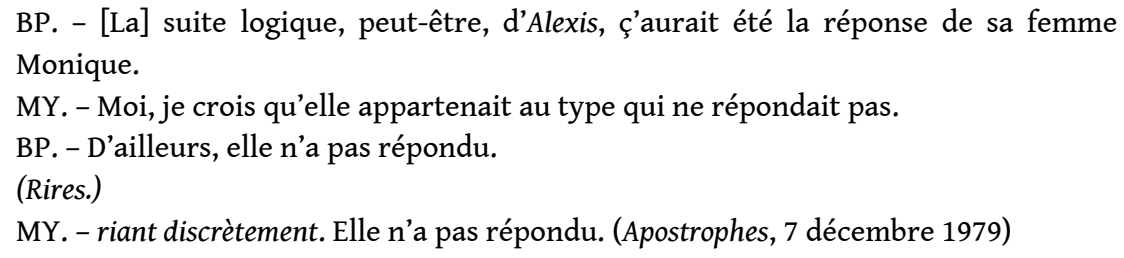

En pénétrant sur le territoire de l'écrivain, celui de l'invention, en donnant corps aux personnages de papier et en en parlant comme d'êtres de chair, Pivot s'emploie à conférer un surcroît d'existence à l'univers fictionnel. D'ailleurs, nombreuses sont ses questions dans lesquelles se superpose, à la demande d'un complément de sens, celle d'un supplément de fable. Dans le même temps, narrant de façon synthétique les récits de ses invités, les étendant dans une nouvelle direction ou vers un prolongement inattendu, Pivot les éclaire d'un nouveau sens, tout particulièrement lorsqu'il s'agit pour lui de rapporter l'univers fictionnel au monde référentiel. 


\subsection{Faire communiquer la fiction et le réel}

Le jeu que Pivot pratique avec l'univers fictionnel ou diégétique de ses invités explique sans doute pour partie les crispations qu'il a pu générer, aussi bien que son succès. La stratégie qu'il adopte consiste à débusquer le réel qui se dissimule sous le voile de la fiction et à rapporter celle-ci à celui-là. Par exemple, dans son entretien avec Albert Cohen :

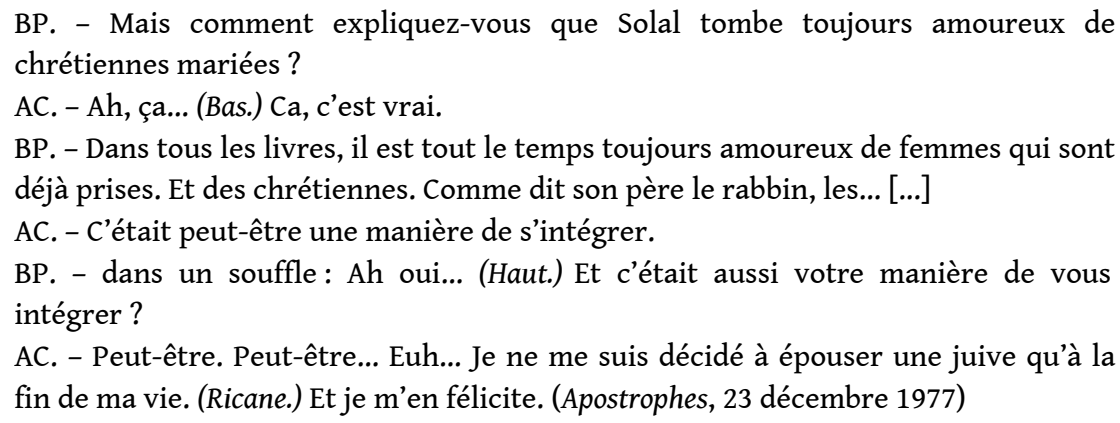

Pivot confère au Cohen octogénaire la gloire donjuanesque que celui-ci a attribuée à son personnage, jusqu'à le rendre aussi sympathique qu'impressionnant aux yeux du public de l'émission. Il obtient de surcroît une révélation d'importance sur la vie du juif émigré, fonctionnaire international, auquel il parvient à faire confesser qu'il aurait " peut-être »- usé de l'amour des femmes comme d'un mode d'intégration.

Somme toute majoritairement mimétique (Charaudeau 1991: 50-66) dans sa façon de procéder, au point de couler ses interventions dans le style d'expression propre à chaque auteur, l'animateur paraît laisser à l'écrivain le soin de donner le ton de l'échange, se chargeant pour sa part d'ordonner le contenu, afin d'obtenir les révélations qui vont constituer les clous de son spectacle. Pivot goûte particulièrement le fait de livrer les «clefs» qui permettent au lecteur d'apprécier les référents réels d'un récit. En témoigne admirablement, dans Bouillon de culture, l'épisode du 24 mars 2000, où il questionne de façon soutenue Dominique Rolin à propos du personnage de Jim dans son Journal amoureux, pendant que le réalisateur et le cadreur, avertis, soulignent les réactions amusées de Philippe Sollers, installé à côté de l'écrivain d'origine belge. Tout à coup, Pivot affirme de but en blanc que Jim est en réalité Sollers et Rolin, décontenancée, ne nie pas, ainsi qu'elle s'en souvient dans ses entretiens avec Patricia Boyer de Latour :

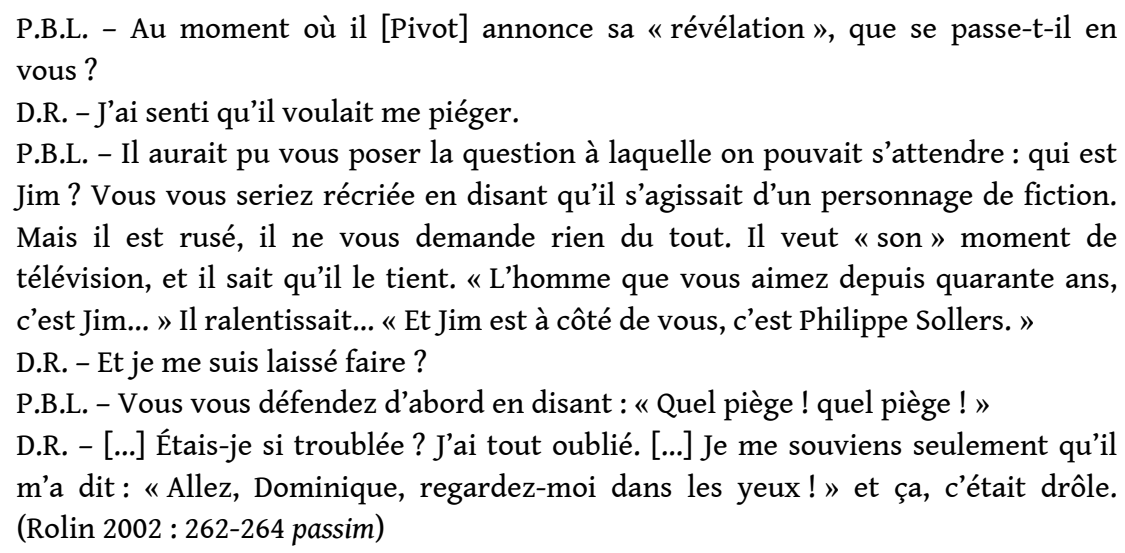

41 Reste que, parfois, Pivot se trompe et l'interprétation tombe à l'eau. Duras, par exemple, ne cesse de déstabiliser son interlocuteur, répondant à côté des questions, 
suivant son propre fil narratif auquel l'intervieweur essaie de nouer ou d'imposer le sien. Lorsqu'il essaie de revenir à ses moutons, Duras ne l'écoute pas ou ne répond qu'avec retard, à un rythme lent qui n'appartient qu'à elle. Pivot se montre désarçonné du rétablissement négatif de la vérité par l'écrivain, à tel point qu'il ne veut y croire et finit par livrer - sans nécessité aucune - l'embryon narratif qu'il avait imaginé en préparant l'émission :

BP. - Et puis, vous racontez qu'elle [la mère de MD] a - je ne sais plus, je crois que c'est dans Un barrage contre le Pacifique - qu'elle a joué pendant dix ans du piano dans un cinéma.

MD. - Oui. C'est pas vrai, ça.

BP. - Ça, c'est pas vrai?

MD. - Non.

BP. - Ah non ? C'est pas vrai ? Alors, euh, oui...

MD. - Elle jouait du piano mais... Oui...

BP. - Ah bon ? Ça, c'est inventé ? C'est parce que moi, j'ai...

MD. - C'est une autre femme.

BP. - Ah oui, d'accord. Moi, j'ai fabulé, je pense que je me suis dit : et alors, elle a joué pendant dix ans, donc elle n'a jamais pu voir le film, puisqu'on jouait pendant que le film passait et je me suis dit donc, tiens, Marguerite Duras, elle est venue au cinéma peut-être pour que sa mère voie ses films. Voilà ce que je me suis dit. Mais finalement, c'est complètement faux. Alors, sur ce portrait qu'on trouve dans Un barrage contre le Pacifique, est-ce qu'il s'applique à votre mère ? (Pivot lit un extrait du roman.) (Apostrophes, 28 septembre 1984)

L'animateur ne se décourage pas lorsque l'une de ses interprétations fait chou blanc, au contraire. La co-construction de l'émission et des entretiens en passe ainsi, d'une manière ou d'une autre, par des moments de contestation qui contribuent à en assurer la dynamique dramatique. Dans ces circonstances, Pivot n'hésite pas à user de signaux agonaux.

BP. - Et est-ce qu'elle [la mère de MD], elle avait honte de votre liaison avec le Chinois?

MD. - après un temps : Elle ne le connaissait pas.

BP. - Comment : « Elle ne le connaissait pas » ? Elle dînait avec vous...

MD. - Oui. Elle dînait avec nous mais elle ne le connaissait, elle ne savait pas ce qui se passait. (Silence.)

BP. - J'y crois pas. J'y crois pas.

MD. - [...] C'est pas peu dire, ce que je vous dis là. [...] Elle ne le savait pas, elle ne l'a jamais su.

BP. - Ça me parait incroyable. (Apostrophes, 28 septembre 1984)

Dans ces conditions, alors que sa fabulation ne fait pas mouche, l'ethos d'intervieweur de Pivot se cristallise dans cette résistance au croire qui fonde la fiction. Sur le plateau, il s'agit en effet pour l'intervieweur de faire sortir la fiction de ses gonds. Là réside sans doute l'une des raisons de l'ambiguïté de ces émissions où la télévision se met au service de la littérature.

\subsection{Un « oncle d'Amérique »}

Pivot fait sienne l'idée de « solidarité de travail intellectuel entre les écrivains [...] et les médias » formulée par Barthes (Pivot 2001: 130). Il considère que les interviews ont repris le rôle jadis dévolu aux préfaces et autres adresses «Au lecteur» qui visaient à orienter l'interprétation de ce dernier. S'il a foi dans le rôle de l'entretien, il n'affiche cependant pas de prétention littéraire, pas même par le biais du statut de critique, dont 
les «lettres de noblesse » le rendent plus proche à ses yeux de l'espace canonique que ne l'est l'intervieweur : «C'est [...] un contresens de faire de moi un critique. D'ailleurs les critiques ne s'y sont pas trompés, ils savent bien que je ne suis pas de leur famille, de la vraie, celle qui a des lettres de noblesse, ou que si j'en suis c'est à titre d'oncle d'Amérique, nouveau riche tapageusement médiatisé » (Pivot $2001: 67$ ). L'animateur d' Apostrophes ne se considère pas (et juge n'être pas considéré) comme étant « de la paroisse » (148), autrement dit du sérail de l'intelligentsia parisienne. Reprenant à son compte une formule de Pierre Nora, Pivot estime que l'intervieweur doit se faire « interprète de la curiosité publique » (33).

Son ethos se déploie alors en retrait par rapport au centre de l'espace littéraire et à la sacralisation dont il fait l'objet, selon un modèle à la fois profane (la noblesse) et religieux (la paroisse). En dépit de l'humilité qui lui tient lieu de marque de fabrique, la figure de Pivot, qui a tiré sa légitimité d'une honnêteté patente, s'est elle-même vu consacrée ${ }^{8}$, au même titre que son émission (51). Premier non-écrivain à siéger à l'Académie Goncourt, sa position lui permet d'élever au rang d'objets sacrés les livres en lesquels il croit, quoique selon des voies paradoxales, puisqu'elles impliquent souvent un geste de profanation. Si Pivot amène le spectateur à investir le territoire de la littérature, franchissant la ligne de démarcation entre la fiction et la réalité, non-lieu intouchable aux yeux de nombre d'écrivains, c'est de façon à rapporter la première à la seconde. Contribuant à la mise en valeur du livre et des auteurs, selon un modèle critique régi par le paradigme beuvien de l'homme et de l'œuvre, cette stratégie explique pour une part le succès de l'émission animée par Bernard Pivot, en même temps que le sentiment de gêne qui a pu s'y attacher pour certains spectateurs.

\section{Conclusion}

S'assignant pour mission de recueillir des informations de la bouche de ses interlocuteurs, l'intervieweur doit faire preuve d'un savoir-faire qui implique, certes, une part d'effacement, mais également la mise en œuvre d'une singularité. Il s'agit pour lui de jouer de la tension impliquée entre ces deux pôles, selon un équilibre parfois précaire avec lequel il faut composer, en fonction des circonstances et des interlocuteurs. À cet égard, la gestion des échanges qui incombe à l'intervieweur suppose notamment le traitement de la rencontre entre des champs discursifs parfois hétérogènes. Pour un intervieweur, interroger un écrivain entraîne inéluctablement de devoir se positionner par rapport au discours littéraire qu'il incarne. En témoignent les pratiques de Madeleine Chapsal, de Jacques Chancel et de Bernard Pivot. Comment négocier une position singulière dans le discours dès lors que l'entretien se tient dans le champ journalistique, dont la spécificité est de se mettre au service du discours qu'il accueille tout en remplissant un cahier des charges propre?

Les différences de style sont manifestes entre ces trois figures médiatiques, autant que celles de leurs positionnements à l'endroit de la littérature et de la figure de l'écrivain. Madeleine Chapsal tient de diverses façons ses distances avec le rôle de journaliste au profit de la constitution d'un ethos où la familiarité avec les écrivains en fait une intervieweuse de choix. Si Jacques Chancel ne sort pas de son rôle d'intervieweur et ne se cantonne pas à recevoir des écrivains, il n'en accorde pas moins une place décisive à la littérature, jusqu'à élever certains de ses interlocuteurs au rang d'écrivains et à faire de ses livres d'entretiens des rencontres de nature littéraire. Enfin, si Bernard Pivot se 
tient à l'écart de l'espace canonique, c'est pour exploiter librement les ressources du discours littéraire, selon une dramaturgie qui, dans le même temps, désacralise et resacralise la littérature et sa part de fiction.

L'un des communs dénominateurs les plus manifestes chez ces trois intervieweurs réside dans un traitement du discours littéraire marqué au coin de la sacralisation. La mobilisation du discours constituant (Maingueneau 2004: 46-49) de la religion pour donner corps au discours littéraire apparaît, dans ce contexte, comme un point de référence majeur dans les stratégies de positionnement de ces intervieweurs. S'il convient de faire la part de l'ironie qui sous-tend leur discours, pour ces éminents praticiens de l'entretien d'écrivain, le discours littéraire ne saurait, de toute évidence, être abordé de façon quelconque, même si son traitement médiatique exige une forme de familiarité, voire de profanation, qui apparaît comme le tribut de la médiatisation qui se noue à l'occasion de l'entretien. Comme l'écrit Madeleine Chapsal dans la postface à Ces voix que j'entends encore : « [u]n écrivain, ça n'est pas n'importe qui, et en même temps ça l'est » $(2011: 359)$.

\section{BIBLIOGRAPHIE}

Amossy, Ruth. 2010. La présentation de soi. Ethos et identité verbale (Paris : P.U.F.)

Bolter, Jay David \& Richard Grusin. 1999. Remediation. Understanding New Media, (Cambridge/ London: The MIT Press)

Brasey, Édouard. 1987. L'effet Pivot (Paris : Ramsay)

Chancel, Jacques. Radioscopie. Entretien avec Albert Naud, 12 novembre 1969 (Paris : France Inter) Chancel, Jacques. Radioscopie. Entretien avec Brigitte Bardot, 7 février 1970 (Paris : France Inter) Chancel, Jacques. Radioscopie. Entretien avec Jacques Monod, 18 novembre 1970 (Paris : France Inter)

Chancel, Jacques. Radioscopie. Entretien avec Jean Bernard, 24 janvier 1973 (Paris : France Inter)

Chancel, Jacques. 1970. Radioscopie, 1 (Paris : Laffont)

Chancel, Jacques. 1971. Radioscopie, 2 (Paris : Laffont)

Chancel, Jacques. 1983. Le livre franc, avec Hubert Nyssen et les invités de "Parenthèses", (Arles : Actes Sud/France Inter)

Chancel, Jacques. 1988. Entretien avec Jean Bernard. Figures, Antenne 2, 28 décembre. Extrait sur le site de l'INA : http://www.ina.fr/video/I08179383 (accès le 15 janvier 2014)

Chapsal, Madeleine. 1959. «Répétitions : Les séquestrés d'Altona ». L’Express, 10 septembre, 27

Chapsal, Madeleine. 1960b. « Prix Nobel. Avant Stockholm ». L’Express, 10 décembre, 49-50

Chapsal, Madeleine. 1962. « Entretien. Truman Capote ». L'Express, 12 avril, 32-33

Chapsal, Madeleine. 1984 [1983]. Envoyez la petite musique (Paris : Le livre de poche) 
Chapsal, Madeleine. 2011. Ces voix que j'entends encore (Paris : Fayard)

Charaudeau, Patrick. 1989. « Le dispositif socio-communicatif des échanges langagiers ». Verbum, $12: 1,13-25$

Charaudeau, Patrick (éd.). 1991. La télévision. Les débats culturels. « Apostrophes » (Paris : Didier Erudition)

Ducas, Sylvie. 2003. «À défaut de génie... : La panthéonisation de Bernard Pivot ». Communication et langages, $135,73-86$

Héron, Pierre-Marie. 2000. Les écrivains à la radio : les Entretiens de Jean Amrouche. (Montpellier : Presses Universitaires de la Méditerranée \& INA)

Kalifa, Dominique et al. (éds.). 2010. La civilisation du journal : histoire culturelle et littéraire de la presse française au XIXe siècle (Paris : Nouveau monde)

Kerbrat-Orecchioni, Catherine. 2005. Le discours en interaction (Paris : Colin)

Maingueneau, Dominique. 2004. Le discours littéraire. Paratopie et scène d'énonciation (Paris : Colin)

Maingueneau, Dominique. 2009. « Auteur et image d'auteur en analyse du discours ", Argumentation et Analyse du Discours, 3 [En ligne], URL : http://aad.revues.org/660

Martens, David \& Meurée, Christophe. À paraître. « Relations (en)tendues. Interférences auctoriales de l'entretien d'écrivain ». Martens, David, Meurée, Christophe \& Willem Guillaume (éds.). L'entretien d'écrivain. Formes et mutations d'un genre hybride (Rennes : Presses universitaires de Rennes)

Martens, David \& Meurée, Christophe. À paraître. « Ceci n'est pas une interview. Littérarité conditionnelle de l'entretien d'écrivain ».

Pivot, Bernard. 2001 [1991]. Le Métier de lire. Réponses à Pierre Nora. D’Apostrophes à Bouillon de culture (Paris : Gallimard)

Pivot, Bernard. 2004. Marguerite Yourcenar. DVD. (Paris : Gallimard-INA)

Pivot, Bernard. 2004. Marguerite Duras. DVD. (Paris : Gallimard-INA)

Pivot, Bernard. 2004. Albert Cohen. DVD. (Paris : Gallimard-INA)

Rodden, John. 2001. Performing the Literary Interview. How Writers Craft Their Public Selves (Lincoln: University of Nebraska Press)

Rolin, Dominique. 2002. Plaisirs. Entretiens avec Patricia Boyer de Latour (Paris : Gallimard)

Seillan, Jean-Marie. 2010. «L’interview ». Kalifa, Dominique, Philippe Regnier, Marie-Ève Thérenty \& Alain Vaillant (éds.), La Civilisation du journal : histoire culturelle et littéraire de la presse française au XIXe siècle (Paris : Nouveau monde), 1025-1040

Thérenty, Marie-Ève. 2007. La Littérature au quotidien. Poétiques journalistiques au XIX siècle (Paris : Seuil)

Yanoshevsky, Galia. 2011. « La co-construction de l'image d'auteur : le cas de l'entretien littéraire ». (A co-construção da imagem do autor: o caso da entrevista literária). Proceedings of the 3rd. International Symposium on Discourse Analysis: Emotions, Ethos, and Argumentation, 1-4 April 2008 (Belo Horizonte (Brazil): Federal University of Minas Gerais), 269-287

Yanoshevsky, Galia. À paraître. « From Publicity to Form of life. On the Literary Nature of the Author's Interview ». Poetics Today 


\section{NOTES}

1. Au sein des études littéraires, la recherche relative à l'entretien a, pour l'essentiel, été jusqu'à présent centrée autour d'écrivains particuliers. Le rôle des intervieweurs a par conséquent fait l'objet d'une attention plus réduite, en dépit de quelques contributions, comme celle de John Rodden, par exemple, qui postule une forme de réciprocité des types de postures entre écrivains et intervieweurs (Rodden 2001). Pour se borner au domaine francophone et à des travaux collectifs, l'on peut également songer au volume dirigé par Pierre-Marie Héron au sujet de Jean Amrouche (Héron 2000) ainsi qu'à la journée d'études Le Renouvellement des formes de la critique littéraire dans l'entre-deux-guerres: "Autour de Frédéric Lefevre (1889-1949), faiseur de littérature", organisée par Fanny Jaffray le 15 mars 2012 à l'Université Paris-Sorbonne.

2. Nous remercions l'auteure d'avoir eu l'amabilité de nous communiquer cet article, que nous traduisons ici.

3. Communication «Les stratégies de légitimation auctoriale dans le champ littéraire ", au colloque À propos de l'auteur, sous la direction de Matthieu Sergier, Marc van Zoggel et Hans Vandevoorde, Université Saint-Louis, Bruxelles, 14 novembre 2012.

4. Un cas de figure tel que l'entretien suggère la nécessité d'inclure dans cet ensemble notionnel une subdivision supplémentaire au sein de l'espace d'étayage. Il s'agirait de distinguer un « espace d'étayage associé » (qui relève du discours de l'auteur et correspond à ce que Dominique Maingueneau désigne comme " espace associé ») et un " espace d'étayage dissocié » (produit par d'autres instances que l'auteur). Cette distinction permettrait de tenir compte de la spécificité énonciative de discours qui n'occupent pas la même position par rapport à l'espace canonique.

5. La page du site de l'INA présentant l'émission (http://www.ina.fr/contenus-editoriaux/ articles-editoriaux/radioscopie) ne précise pas la date de son interruption définitive.

6. Plusieurs années plus tard, dans l'émission de télévision Figures du 28 décembre 1988, Chancel récidive : «On s'aperçoit, Professeur Jean Bernard, que vous avez eu une vie très riche, sur le plan médical, que vous avez obtenu beaucoup de succès dans vos recherches et que vous avez une crédibilité partout dans le monde et que, malgré tout cela, il y a quand même dans votre vie, comme... je ne sais pas... comme une nostalgie. Il y a un rendez-vous manqué, un rendez-vous manqué avec l'écriture. [...] Vous auriez voulu être un écrivain ». Réponse de Jean Bernard : «Je ne sais pas trop ", à quoi Chancel réplique par un éloquent « Si... », en appuyant longuement sur le « $\mathrm{i} »$. (L'extrait est disponible sur le site de l'INA : http://www.ina.fr/video/I08179383).

7. Lors de l'émission du 18 mars 1978, par exemple.

8. En témoignent les rééditions en DVD de ces émissions pourtant liées à une actualité culturelle ancienne.

\section{RÉSUMÉS}

Fondé sur une interaction verbale entre deux interlocuteurs au moins, l'entretien implique souvent la rencontre entre des champs discursifs différents. De ce point de vue, les acteurs de l'échange, aussi bien l'intervieweur que l'interviewé, sont tenus de se positionner en fonction de l'hétérogénéité discursive sur laquelle se fonde ce type de dialogue. Pour des intervieweurs culturels comme Madeleine Chapsal, Jacques Chancel et Bernard Pivot, respectivement actifs dans la presse écrite, à la radio et à la télévision, la négociation de l'interaction entre discours journalistique et discours littéraire constitue un enjeu central. Le propos de cet article est 
d'analyser les stratégies de positionnement de chacun de ces intervieweurs, lorsqu'ils se trouvent confrontés au champ discursif auquel appartiennent les écrivains.

Based on a verbal interaction between at least two speakers, the interview frequently involves an encounter between different fields of discourse. From this perspective, the interview appears to be the place where the interlocutors are required to position themselves in order to deal with the discursive heterogeneity produced by this kind of dialogue. For interviewers like Madeleine Chapsal, Jacques Chancel, and Bernard Pivot, respectively working in written press, radio and on television, the negotiation of the interaction between journalistic and literary discourse is an important issue. This article aims to analyze positioning strategies of those interviewers as they are confronted with discourse on literature.

\section{INDEX}

Keywords : discourse field, discursive heterogeneity, interview, literature, positioning, press, radio, television

Mots-clés : champs discursifs, entretien, hétérogénéité discursive, interview, littérature, positionnement, presse, radio, télévision

\section{AUTEURS}

\section{DAVID MARTENS}

Université de Louvain (KU Leuven) - MDRN

\section{CHRISTOPHE MEURÉE}

Fonds de la Recherche Scientifique (FNRS) - Université de Louvain, Louvain-la-Neuve (UCL) 